\title{
Changes in ESCRT-III filament geometry drive membrane remodelling and fission in silico
}

\author{
Lena Harker-Kirschneck ${ }^{1,2}$, Buzz Baum ${ }^{2,3 \dagger}$ and Andela Šarić ${ }^{1,2^{*} \dagger}$ (1)
}

\begin{abstract}
Background: ESCRT-III is a membrane remodelling filament with the unique ability to cut membranes from the inside of the membrane neck. It is essential for the final stage of cell division, the formation of vesicles, the release of viruses, and membrane repair. Distinct from other cytoskeletal filaments, ESCRT-III filaments do not consume energy themselves, but work in conjunction with another ATP-consuming complex. Despite rapid progress in describing the cell biology of ESCRT-III, we lack an understanding of the physical mechanisms behind its force production and membrane remodelling.

Results: Here we present a minimal coarse-grained model that captures all the experimentally reported cases of ESCRT-III driven membrane sculpting, including the formation of downward and upward cones and tubules. This model suggests that a change in the geometry of membrane bound ESCRT-III filaments - from a flat spiral to a 3D helix-drives membrane deformation. We then show that such repetitive filament geometry transitions can induce the fission of cargo-containing vesicles.

Conclusions: Our model provides a general physical mechanism that explains the full range of ESCRT-III-dependent membrane remodelling and scission events observed in cells. This mechanism for filament force production is distinct from the mechanisms described for other cytoskeletal elements discovered so far. The mechanistic principles revealed here suggest new ways of manipulating ESCRT-III-driven processes in cells and could be used to guide the engineering of synthetic membrane-sculpting systems.
\end{abstract}

Keywords: ESCRT-III, Membrane remodelling, Membrane scission, Computer simulations, Biological physics

\section{Introduction}

Cellular membranes require constant remodelling to allow cells to maintain homeostasis, to grow, and to divide. This involves protein machines that can physically sculpt membranes in both directions, toward and away from the cytoplasm. The ESCRT-III family of proteins (endosomal sorting complexes required for transport III) is the only cellular apparatus known to deform and cut cell membranes protruding away from the cytoplasm. This is a topologically difficult transition, as the membrane needs

\footnotetext{
*Correspondence: a.saric@ucl.ac.uk

†Buzz Baum and Andela Šarić contributed equally to this work.

'Department of Physics \& Astronomy, University College London, Gower Street, London, WC1E 6BT UK

${ }^{2}$ Institute for the Physics of Living Systems, University College London, Gower Street, London, WC1E 6BT UK

Full list of author information is available at the end of the article
}

to be deformed from the inner side of the membrane neck. ESCRT-III proteins perform this task by forming spiral/helical filaments that associate with the cytoplasmic face of biological membranes [1-4]. This enables them to perform a wide range of membrane sculpting and snipping processes from archaeal to eukaryotic cells, such as cytokinesis [5, 6], multi-vesicular body formation [7-9], virus release [10,11], membrane repair [12], and nuclear envelope re-sealing [13]. Despite many attempts to use physical principles to explain how ESCRT-III performs these functions[14-17], it is not clear how a single protein machine has the versatility to carry out this full range of functions.

While a recent model of ESCRT-III filaments as spiral springs $[14,18]$ offers a simple way to link ESCRT-III polymer formation to membrane deformation, it is unable to

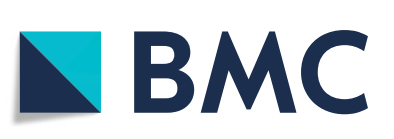

(c) The Author(s). 2019 Open Access This article is distributed under the terms of the Creative Commons Attribution 4.0 International License (http://creativecommons.org/licenses/by/4.0/), which permits unrestricted use, distribution, and reproduction in any medium, provided you give appropriate credit to the original author(s) and the source, provide a link to the Creative Commons license, and indicate if changes were made. The Creative Commons Public Domain Dedication waiver (http://creativecommons.org/publicdomain/zero/1.0/) applies to the data made available in this article, unless otherwise stated. 
explain: (i) the sign of the deformation-spiral tension can be released in both upwards and downwards directions; (ii) membrane scission; (iii) the role of energy consumption via Vps4 ATPase in ESCRT-III function; and (iv) the ability of ESCRT-III to deform both flat and curved membranes. Here, we have used coarse-grained molecular dynamics simulations to develop the first particlebased model of ESCRT-III filament function. Strikingly, our model suggests that a single filament geometry cannot explain the experimentally observed behaviours and that membrane shaping and topological transitions require energy-dependent transitions in chiral filament geometry.

\section{Results}

\section{Coarse-grained model}

Our nanoscale ESCRT-III filament model is built of beads connected by springs. The minimal unit required to construct a chiral filament that preserves its flat spiral shape was found to be a triplet of beads, where the beads of neighbouring triplets are interconnected as shown in Fig. 1a. Filament geometry is controlled by bond lengths linking neighbouring triplets (Fig. 1a), while rigidity, measured by filament persistence, is controlled by bond strength between the triplets. Since building units and bond lengths between neighbouring units are equal, the target geometry of such a filament is a closed ring of radius $R$. Though some ESCRT-III filaments are observed as rings [1, 18-20], the effects of volume-exclusion will force longer filaments to form spirals with the adoption of non-preferred curvatures causing a build-up of tension in the filament. The membrane is described using a coarse-grained one particle thick membrane model that reproduces the fluidity and correct mechanical properties of biological membranes [21]. As a check, we confirmed that the main results hold when using a threebead-per-lipid membrane model that explicitly accounts for the existence of the bilayer structure [22] (Additional file 1: Figure S5). Finally, the membrane-filament interface is modelled using a short-range attractive potential between two beads of the triplet (coloured in blue) and the membrane beads. This potential describes the adsorption of the filament onto the membrane, including screened charge-driven adsorption. Further details of the model are described the Additional file 1: Supplementary Information [23-28].

\section{Single filament geometry cannot deform membranes}

Pre-assembled planar ESCRT-III spirals (Fig. 1b) were placed on an equilibrated flat membrane, and their behaviour followed over time as tension in the system was allowed to relax (Fig. 1c). Since the outer arms of the spiral are stretched beyond the preferred filament curvature, while the inner arms are compressed, the filament will attempt to reach mechanical equilibrium by contracting its outer and expanding its inner filament arms, as previously suggested $[14,18]$. This leads to the formation of dense spirals, since the filament cannot overlap with itself. If the modelled filament was allowed to overlap with itself, it would take on a ring shape of the target curvature (Additional File 1: Figure S3). Remarkably, for any tested

\section{FLAT STATE}


Fig. 1. Model development. a Filaments are built out of interconnected three-beaded units. The target geometry of the filament is a flat ring whose radius is determined by the rest lengths of bonds between the triplet units. The inset highlights two neighbouring units connected by 9 bonds to preserve the spiral chirality. The blue beads of the triplet are attracted to the membrane. $\mathbf{b}$ If the filament is longer than the circumference of the target ring, it will acquire a geometry of a spiral with tense bonds, which is our initial configuration of the system. c Placing a flat spiral on the membrane does not lead to a significant membrane deformation. The filament density increases, but its membrane-attracted face remains trapped in the same 2D plane. A shallow buckle that develops around $-5 \mathrm{~nm}$ is due to the membrane enveloping the filament to maximise their contact surface. The arrows highlight simulation snapshots at specific time steps. The radius of the target geometry is $R=20.4 \mathrm{~nm}$ and the persistence length of the filament $l_{\mathrm{p}}=1.8 \cdot 10^{3} \mathrm{~nm}$ 
spiral geometry or level of stored tension, spirals remained effectively flat on the membrane (Additional file 2: Video 1 ), even when all three beads of the filament triplets were allowed to bind to the membrane. Thus, under our model, a tense filament that only possesses in-membrane-plane curvature is not sufficient to drive membrane deformation. In line with this, in vitro experiments have reported ESCRT-III spirals can grow to several hundred nanometers in radius $[18,29]$, without deforming the membrane on which they sit $[1,30]$.

\section{Filament geometry changes}

ESCRT-III filaments have also been observed in a variety of 3D shapes, such as helices and cones [1, 16, 19, 30-34], indicating that ESCRT-III filaments can assume alternative target geometries. To account for this, we allowed our filaments to switch between two well-defined categories of geometrical states. In the first category, the target geometry is a ring that has its membrane binding site lying in a $2 \mathrm{D}$ plane, leading to the formation of spirals when bound to a membrane. In the second, the target geometry switches to a ring with its membrane binding site globally rotated outwards assuming a tilt angle $\tau$ (Fig. 2a). As a result, the membrane attachment site now sits on a $3 \mathrm{D}$ cone/tubule surface, which causes the filament take on a 3D helical shape (Fig. 2a). We suggest that this internal filament tilt, which has not been taken into account in previous models [1416], drives membrane deformation. When a planar spiralshaped filament is placed on a flat membrane and the target geometry is switched through-out the filament to a Ştilted $\check{T}$ state, the relaxation of the filament induces a membrane buckle. This deformation develops away from the cytoplasm and grows in time to a fixed depth (Fig. 2b, Additional file 3: Video 2).
This works as follows. The membrane deformation is initiated by the filament internal tilt which transforms the filament-membrane attraction site from a $2 \mathrm{D}$ plane to a $3 \mathrm{D}$ conical surface with aperture $\theta=90^{\circ}-\tau$. This initiates an out-of-plane membrane deformation, which frees the filament from being trapped in a $2 \mathrm{D}$ plane, allowing the filament arms to move in 3D and relax closer to their desired radius $R$. While doing so, the outer filament arms push the inner arms down into the buckle, deepening the deformation. Because this is achieved via volume exclusion, tension in the filament only makes an indirect contribution to membrane deformation by encouraging the filament to enter the buckle (see Additional file 1: Figure S3 and Additional file 4: Video 3 and Additional file 5: Video 4 for control experiments). The resulting filament assumes a tightly coiled helical geometry, even though the filament does not possess a preferred target pitch.

While helical filaments will attempt to relax into a state in which neighbouring rings are stacked and tilted by $\tau$, the wrapping of membrane about each separate ring of the spiral in the tilted state is not energetically favourable. The trade-off deformation is therefore a cone. Only for filament's internal tilt of $\tau=90^{\circ}$ do we observe tubule formation (Fig. 3a).

\section{Quantifying membrane deformations}

The shape of the membrane deformation is determined largely by the filament tilt. This is shown in Fig. 3b, where the depth and the sign of the deformation are found to depend on the tilt angle and the radius of the tilted state. Strikingly, a single model parameter-the tilt angle $\mathrm{T}$ generates a large variety of observed states ranging from flat spirals, to conical helices of varying aperture and even tubules in both directions.

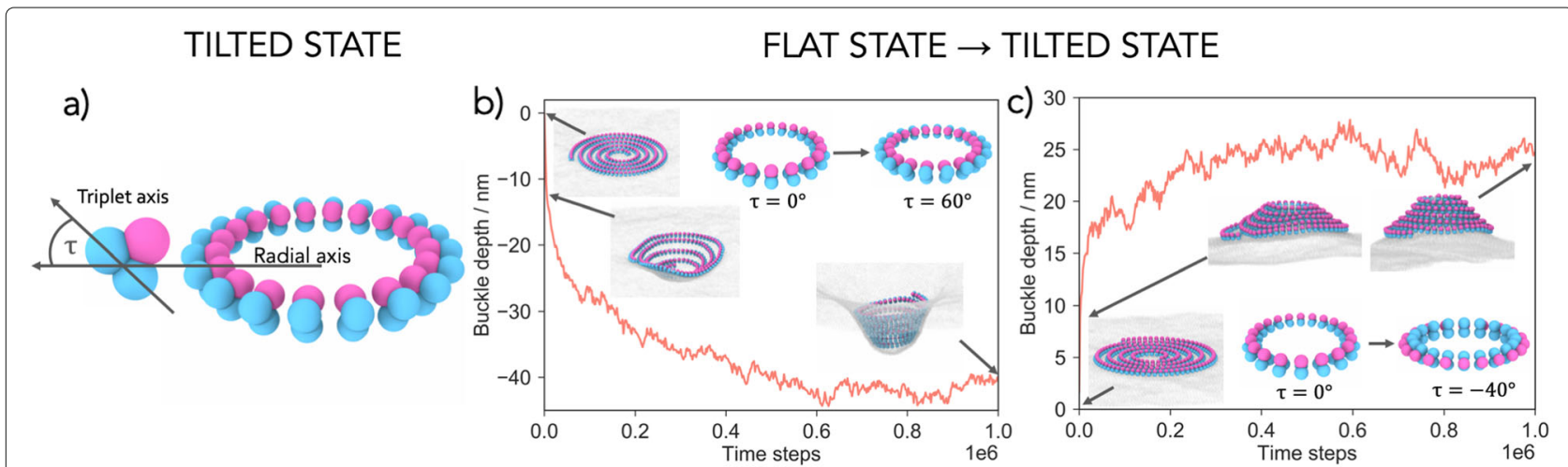

Fig. 2. Transition between filament geometries creates membrane deformation. $\mathbf{a}$ The tilted state is defined by an angle $\tau$, which is the angle between the radial axis and the triplet subunit axis. $\mathbf{b}$ Rotating the target geometry ring by a tilt angle $\tau$ ( $\tau=60^{\circ}$, depicted in the inset) creates downward membrane deformations, away from the cytoplasm. The curve shows the depth of the deformation over time $(R=15.3 \mathrm{~nm}$ and $\left.I_{p}=1.8 \cdot 10^{3} \mathrm{~nm}\right)$. c The direction of the deformation can be reversed by tilting the filament outwards, rather than inwards. The new target geometry is now a ring with $\tau=-40^{\circ}, R=11.5 \mathrm{~nm}$, and $/ \mathrm{p}=1.8 \cdot 10^{3} \mathrm{~nm}$. The underlying curve shows the height of the developing deformation over time 

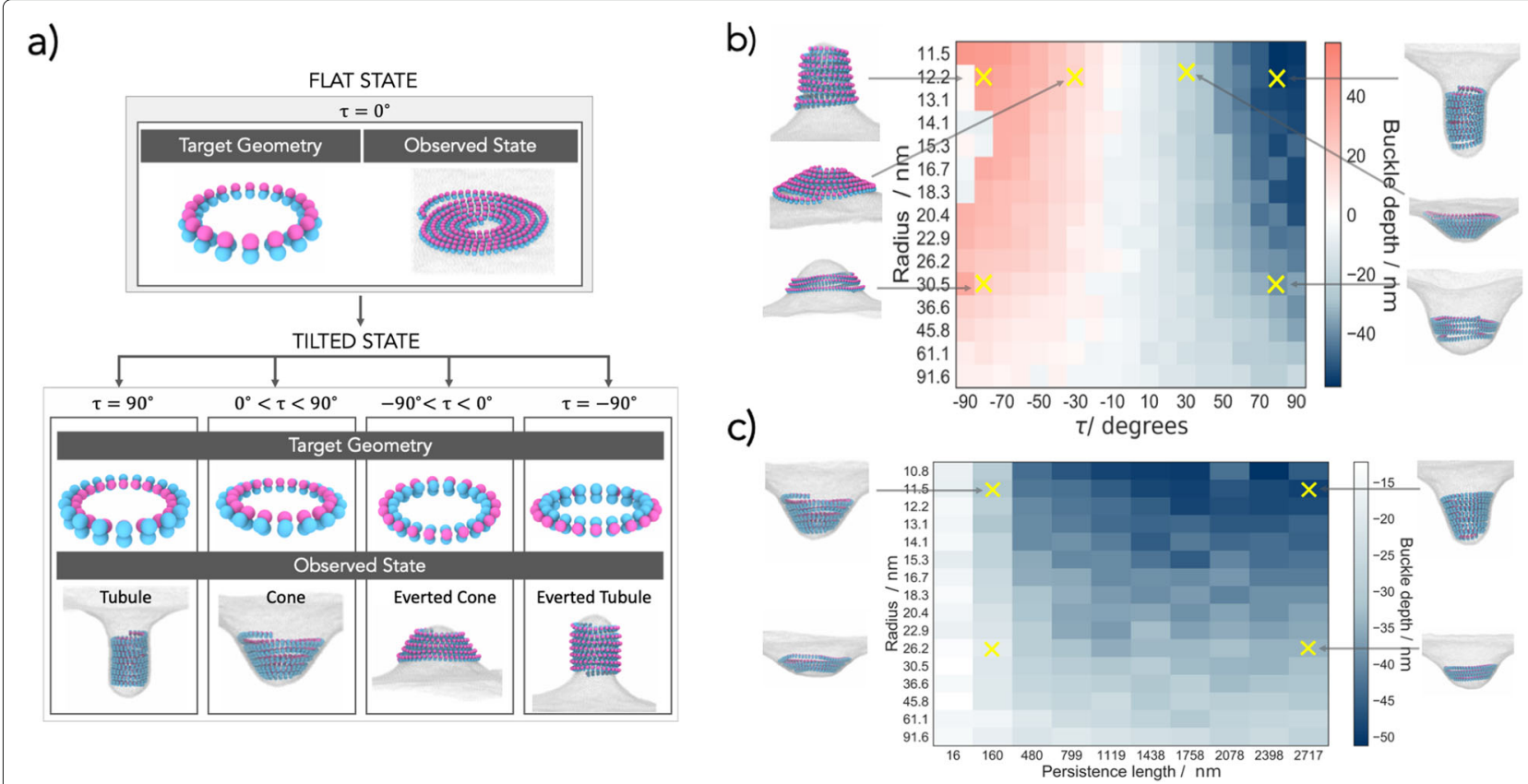

Fig. 3. The filament tilt determines the shape and sign of the membrane deformation. Overview of the two-state model, providing examples for different outcomes. $\mathbf{a}$ In the flat state, the target geometry is a planar ring with the membrane attraction sites facing downwards $\left(\tau=0^{\circ}\right)$ and the filament is observed as a flat spiral. By internally rotating the target geometry ring, we enter the tilted state in which tubules develop for $\tau=90^{\circ}$, cones for $0^{\circ}<\tau<90^{\circ}$, everted cones for $\tau<0^{\circ}$, and everted tubules for $\tau=-90^{\circ}$. $\mathbf{b}$ The buckle depth dependence on the target radius $R$ and the angle $\tau$ of the tilted state. Each simulation started off with the same initial spiral in the flat state with persistence length $/ \mathrm{p}=1.8 \cdot 10^{3} \mathrm{~nm}$. $\mathbf{c}$ The buckle depth dependence on the target radius $R$ and the persistence length $/ \mathrm{p}$ of the tilted state $\left(\tau=60^{\circ}\right)$. Each simulation started off with the same initial spiral in flat state. The insets show snapshots of the representative cases

The depth of the deformation is also influenced by the preferred filament radius (Fig. 3b) and filament rigidity (Fig. 3c). Since all the filaments in our simulations have the same total length, filaments that have larger preferred radii tend to result in shorter helices that give rise to shallower deformations. The persistence length functions here as a measure of the amount of intrinsic tension that the filament possesses. Naturally, filaments with small persistent lengths do not have a strong drive to achieve their target geometry and cannot deform the membrane (Fig. 3c). For increasing filament stiffness, deeper buckles develop, and their shape changes from conical toward tubular. The internal filament energy now dominates over the elastic energy required to bend the membrane, and a tubular deformation that accommodates the preferred filament radius is formed. Finally, to test the possible role of the helical pitch, which is not implemented in our model, we also carried out simulations that included an explicit helical pitch (see Additional file 1: Supplementary Information). Interestingly, we found that this preferred pitch does not influence the resulting deformations and that the filament always remains in a tightly coiled helical state when deforming the membrane (Additional file 1: Figure S4).
The model also provides a simple and intuitive explanation for the origin of the symmetry breaking in the membrane deformation. The buckling direction is determined by the sign of the tilt angle $\tau$, i.e. by the 3D chirality in the filament. We should therefore be able to reverse the buckle direction simply by reversing the filament tilt. As can be seen in Figs. 2c and 3b, by moving the membranebinding sites to the inside of the filament buckles can be induced in the opposite direction, toward the cytoplasm (Additional file 6: Video 5). In line with this possibility, Cullough et al. reported the formation of both upward and downward buckles of ESCRT-III filaments, depending on the filament composition[3]. Hence, it is possible for ESCRT-III filaments to induce different types of membrane deformation depending on whether they form a helix or an inverted helix.

Interestingly, in our model, there is a bias in the system that leads to a preference for downward buckles (as can be seen in Fig. 3b). Simulations for large negative tilt angles $\left(\tau=-80^{\circ}\right.$ to $\left.-90^{\circ}\right)$ do not show any deformation, an anomaly that is not mirrored for positive tilt angles. The reason for this bias in the energy landscape is that, for an upward buckle to form, the membrane must adhere to the filament from "the inside" and must adopt 
a larger curvature than a downward membrane deformation caused by the same amount of filament rotation, rendering upward deformations more costly. In the case of downward deformations, the membrane envelops the filament from the outside, resulting in a smaller curvature, reducing the amount of energy required to deform the membrane. These observations may explain why cytosolic ESCRT-III filaments preferentially deform and cut membranes away from the cytoplasm.

\section{Membrane scission}

To explore whether transitions in filament geometry can also drive scission, we introduced a simple generic cargo into the model. This cargo is allowed to weakly adhere to the membrane (see Additional File 1: Supplementary Information for details) so that it remains adsorbed and creates a shallow deformation. The adhesion is, however, too weak to cause substantial cargo wrapping by the membrane and spontaneous budding. We then polymerise a flat ESCRT-III spiral around the cargo. This models the way the ESCRT system is thought to corral cargo in cells [35]. Under these conditions, a switch in filament geometry induced by a transition to a positively tilted state initiates a conical membrane deformation, which traps the cargo at its centre underneath the ESCRT-III helix, as shown in Fig. 4a. In this configuration, the filament stabilises the energetically costly membrane neck. If the filament geometry reverts back to a flat state, this destabilises the neck and causes membrane scission, releasing a membrane bud that carries the cargo particle, while the filament retracts back to the cytoplasm (Additional file 7:
Video 6). Thus, transitions between distinct geometrical states can drive ESCRT-III-mediated membrane scission to mimic the role of ESCRT-III in the formation of vesicles.

The same type of repetitive changes in filament geometry can also sever membranes in situations in which the cargo itself induces substantial membrane deformation, as observed in the case of Gag-driven budding of HIV1 [36]. Figure $4 \mathrm{~b}$ shows the scenario in which we used a larger concentration of membrane receptors such that the cargo binds to the membrane strongly enough to cause a substantial membrane deformation, but still not strongly enough to bud off on its own. In this case, the helical filament geometry is needed for the filament to enter the membrane neck, while the change from helical to flat again performs the membrane scission (Additional file 8: Video 7).

\section{Discussion}

This minimal coarse-grained model of ESCRT-III filaments in contact with lipid membranes captures many of the experimentally observed behaviours of this versatile membrane apparatus, including different filament morphologies, diverse membrane deformations, scission, and cargo-loaded membrane budding and ESCRT recycling [37]. The key ingredient of the model is the transition of the filament between two different geometrical states-a flat one, where the membrane-binding sites of the filament lie on a flat plane inhibiting membrane deformation, and a helical one, where the membrane-binding sites shift into a $3 \mathrm{D}$ surface.

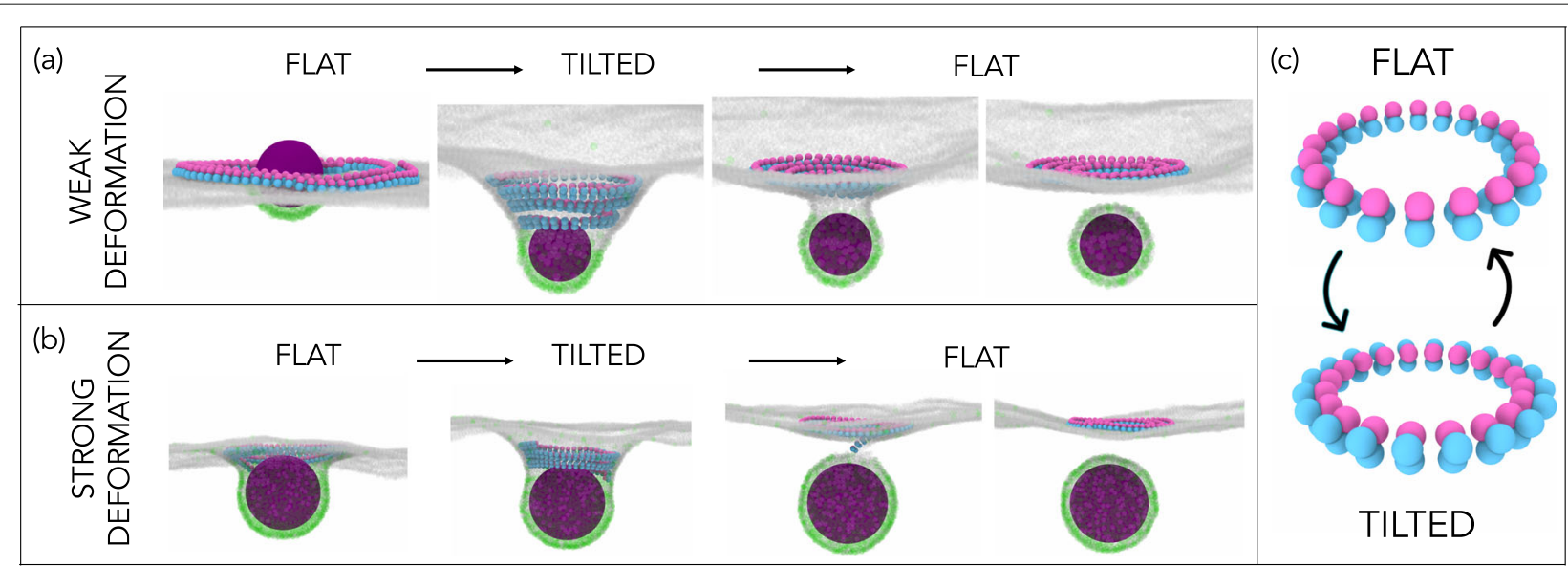

Fig. 4. Repetitive filament transitions can sever membranes. a A filament is polymerised around a cargo (magenta sphere) that weakly binds to the dark receptors in the membrane but cannot bud off on its own. Switching from a flat $\left(R=14.1 \mathrm{~nm}, \tau=0^{\circ}\right)$ to a tilted state $\left(R=14.1 \mathrm{~nm}, \tau=60^{\circ}\right)$ causes the membrane deformation. Switching back from the tilted to the flat state causes cargo budding where the filament is released back to the cytoplasm. b The repetitive filament geometry changes also drive membrane scission in the case where the cargo has already created a substantial membrane deformation on its own, achieved by using a higher density of membrane receptors. Here the filament change from flat to helical enables the filament to enter the membrane neck and deepen it, while the opposite geometry change again performs the scission. The filament parameters and simulation protocol are the same as in a. c Target geometries of the flat and tilted states 
How might the different geometrical states be achieved in the context of ESCRT-III functions in vivo? We suggest that the presence of different members of the ESCRT-III family of proteins in copolymers $[3,4,31,38]$ may control the overall geometrical state of the filament. The action of Vps4 ATPase may then remodel the filament, for example by preferentially removing a specific ESCRTIII protein from the copolymer, to generate a filament that has an altered geometry. In doing so the system can produce mechanical work.

Our suggestion fits with the experimental evidence that binding partners of ESCRT-III can change filament structure [3, 39-41] and transform flat spiral filaments into helices $[1,30]$. The large variety of ESCRT-III binding partners may enable ESCRT-III filaments to sample a wide variety of target geometries depending on the copolymer composition, thereby facilitating diverse ESCRT functions on very different scales and topologies. Further changes in the composition of the filament through the action of the Vps4 ATPase, which has been shown [30, 42] to induce filament depolymerisation/turnover, would change the internal structure of the filament toward another target geometry, leading to membrane scission. We expect that similar geometrical transitions between or even within the geometry states may also enable ESCRT-III filaments to function in membrane healing and cell division. Local rather than global transitions can even lead to mixed geometry filaments that are flat on the outside and helical in the centre [3].

Our model makes a strong prediction that the geometry transition from spiral to helical is needed for ESCRTIII function. Indeed, ESCRT-III (co)filaments have been observed in planar forms and with helical conformations in solution and in cryo-EM [1, 3, 31, 37]. We believe that a functional role for this structural transition could be confirmed, e.g. by using cryo-EM to image structures of filaments caught during or at the end of membrane deformation. While previous physical models of ESCRTIII function [14-17] have not included any energy input, our model suggests that the role of Vps4 ATPase likely lies in inducing a switch in the filament geometry. This analysis aligns well with the recent experiments by Goliand et al., in which the geometry change between a ring and a spiral ESCRT-III filament, caused by Vps4, is suggested to drive the constriction of the intercellular membrane bridge between two dividing cells [33]. Similarly, Maity et al. have recently shown that Vps4 causes changes in the helical radius of ESCRT-III helical filaments in vitro, again suggesting that a change in filament geometry underlies ESCRT-III-mediated membrane remodelling [43]. Finally, Pfitzner et al. have recently demonstrated that Vps4 ATPase promotes sequential changes in the composition of various ESCRT-III proteins within the filament, which is directly coupled to the filament's ability to remodel the membrane [34]. Hence, the multiple filament geometry changes proposed by our model might be caused by an intricate interplay between the Vps4 ATPase and different ESCRT binding partners.

It is also important to discuss some limitations of our model. The model is coarse-grained in nature and does not capture structural details of monomers within the spiral, but only the global chiral structure of the filament as a whole. As such, a filament in our study can also represent a co-polymer made of two or more different monomer types, so we cannot use this model to make predictions about filament substructure. Our simulations were performed by applying global geometry changes to pre-assembled filaments. This enabled us to probe general mechanisms of ESCRT-III action; however, to capture the full detailed mechanism, it will be crucial to include dynamic polymerisation and depolymerisation of the filament and local changes in the geometry. This will be the topic of our future studies.

In summary, this general physical model captures a novel non-equilibrium mechanism of membrane remodelling by elastic filaments as they undergo a global change in geometry. In our simulations, the energy required to drive these transitions is effectively supplied into the system by altering the filament geometry, which mimics the changes in the filament chemical composition through binding other ESCRT components or Vps4 ATPase. This coupling between the chemical composition and filament geometry produces mechanical work with which membranes can be deformed and cut. Beyond its contribution to the understanding of the ESCRT-III apparatus and membrane remodelling, our model also opens a range of possibilities for studies of membrane physics during energy-driven processes. Our results also suggest a novel way of controlling membrane remodelling in synthetic systems, applicable for instance to membrane deformation by self-assembled DNA origami structures [44], where geometrical transition of the systems should be relatively straightforward to control.

\section{Supplementary information}

Supplementary information accompanies this paper at https://doi.org/10.1186/s12915-019-0700-2.

Additional file 1: Contains details on the computer model and simulation set-up (Section I), the supporting results on filament geometry when volume exclusion is turned off (Section II A and B), and the supporting results with an explicit bilayer membrane model (Section II C).

Additional file 2: A polymer spiral in the flat state does not lead to a significant membrane deformation. The filament density increases, but its membrane-attracted face remains trapped in the same 2D plane (see also Fig. 1c).

Additional file 3: Switching from the flat to the tilted a ring rotated by a tilt angle $\tau\left(\tau=60^{\circ}\right)$ creates downward membrane deformations, away from the cytoplasm (see also Fig. 2b). 
Additional file 4: Self-overlapping filament spiral in the flat state ends up in a flat ring conformation (see also Figure S3).

Additional file 5: Self-overlapping filament spiral in the tilted state ends up in a tilted ring conformation (see also Figure S3).

Additional file 6: Switching from the flat to tilted ring $\left(\tau=-40^{\circ}\right)$ creates upward membrane deformations, toward from the cytoplasm (see also Fig. 2c).

Additional file 7: A cargo particle is corralled on the membrane by the spiral ESCRT-III polymer in the flat state. The cargo binds to the dark gray receptors in the membrane but cannot bud off on its own. Switching the filament geometry from a flat to a tilted state $\left(\tau=60^{\circ}\right)$ deforms the membrane, while switching back from the tilted to the flat state causes cargo budding where the filament is released back to the cytoplasm (see also Fig. 4a).

Additional file 8: A cargo particle creates deep membrane deformation on its own, due to the high percentage of membrane receptors. Switching the filament geometry from a flat $\left(\tau=0^{\circ}\right)$ to a tilted state $\left(\tau=60^{\circ}\right)$ enables the filament to enter the preformed membrane neck, deepening and stabilising it. Switching back to the flat filament state causes cargo budding where the filament is released back to the cytoplasm (see also Fig. 4b).

\section{Acknowledgements}

We thank Jeremy Carlton, Mike Staddon, Geraint Harker, and the Wellcome Trust Consortium "Archaeal Origins of Eukaryotic Cell Organisation" for fruitful conversations. We thank Peter Wirnsberger and Tine Curk for discussions about the membrane model implementation.

\section{Authors' contributions}

$B B$ and $A S ̌$ conceived and supervised the research. LHK performed the computational experiments and data analysis. All authors participated in the model development, data interpretation, and writing of the manuscript. All authors read and approved the final manuscript.

\section{Funding}

We acknowledge funding from BBSRC LIDo DTP (L.H.K.), the Wellcome Trust (203276/Z/16/Z) and BBSRC (BB/K009001/1) (B.B.), and the Royal Society (UF160266) (A.S.). We are grateful to the UK Materials and Molecular Modelling Hub for computational resources, which is partially funded by EPSRC (EP/P020194/1).

\section{Availability of data and materials}

The datasets supporting the conclusions of this article are available from the UCL data repository https://doi.org/10.5522/04/9804494.v1. The simulations input and configurational files are available from the authors upon request.

\section{Ethics approval and consent to participate}

Not applicable.

\section{Consent for publication}

Not applicable.

\section{Competing interests}

The authors declare that they have no competing interests.

\section{Author details}

${ }^{1}$ Department of Physics \& Astronomy, University College London, Gower Street, London, WC1E 6BT UK. ${ }^{2}$ Institute for the Physics of Living Systems, University College London, Gower Street, London, WC1E 6BT UK. ${ }^{3}$ MRC Laboratory for Molecular Cell Biology, University College London, Gower Street, London, WC1E 6BT UK.

\section{Received: 21 August 2019 Accepted: 17 September 2019}

Published online: 22 October 2019

\section{References}

1. Henne WM, Buchkovich NJ, Zhao Y, Emr SD. The endosomal sorting complex ESCRT-II mediates the assembly and architecture of ESCRT-III helices. Cell. 2012;151(2):356-71.
2. Cashikar AG, Shim S, Roth R, Maldazys MR, Heuser JE, Hanson PI. Structure of cellular ESCRT-III spirals and their relationship to HIV budding Elife. 2014;3:e02184.

3. McCullough J, Clippinger AK, Talledge N, Skowyra ML, Saunders MG, Naismith TV, et al. Structure and membrane remodeling activity of ESCRT-III helical polymers. Sci (NY). 2015;350(6267):1548-51.

4. Effantin G, Dordor A, Sandrin V, Martinelli N, Sundquist WI, Schoehn G, et al. ESCRT-III CHMP2A and CHMP3 form variable helical polymers in vitro and act synergistically during HIV-1 budding. Cell Microbiol. 2013;15(2): 213-26.

5. Carlton JG, Martin-Serrano J. Parallels Between cytokinesis and retroviral budding: a role for the ESCRT machinery. Science. 2007;316(5833): 1908-12. Available from: http://science.sciencemag.org/content/316/ 5833/1908.

6. Morita E, Sandrin V, Chung HY, Morham SG, Gygi SP, Rodesch CK, et al. Human ESCRT and ALIX proteins interact with proteins of the midbody and function in cytokinesis. EMBO J. 2007;26(19):4215-27. Available from: http://emboj.embopress.org/content/26/19/4215.

7. Williams RL, Urbé S. The emerging shape of the ESCRT machinery. Nat Rev Mol Cell Biol. 2007;8:355. EP -

8. Wollert T, Wunder C, Lippincott-Schwartz J, Hurley JH. Membrane scission by the ESCRT-III complex. Nature. 2009;458(7235):172-7.

9. Adell MAY, Vogel GF, Pakdel M, Müller M, Lindner H, Hess MW, et al. Coordinated binding of Vps4 to ESCRT-III drives membrane neck constriction during MVB vesicle formation. J Cell Biol. 2014;205(1):33-49.

10. von Schwedler UK, Stuchell M, Müller B, Ward DM, Chung HY, Morita E, et al. The protein network of HIV budding. Cell. 2003;114(6):701-13. Available from: http://www.sciencedirect.com/science/article/pii/ S0092867403007141.

11. Bieniasz PD. Late budding domains and host proteins in enveloped virus release. Virology. 2006;344(1):55-63. Virology 50th Anniversary Issue. Available from: http://www.sciencedirect.com/science/article/pii/ S0042682205005994.

12. Jimenez AJ, Maiuri P, Lafaurie-Janvore J, Divoux S, Piel M, Perez F. ESCRT machinery is required for plasma membrane repair. Science. 2014;343(6174):1247136.

13. Stoten $C L$, Carlton JG. ESCRT-dependent control of membrane remodelling during cell division. In: Seminars in cell \& developmental biology. vol. 74. Elsevier; 2018. p. 50-65.

14. Lenz M, Crow DJG, Joanny JFmc. Membrane buckling induced by curved filaments. Phys Rev Lett. 2009;103:038101. Available from: https://link.aps. org/doi/10.1103/PhysRevLett.103.038101.

15. Fabrikant G, Lata S, Riches JD, Briggs JA, Weissenhorn W, Kozlov MM. Computational model of membrane fission catalyzed by ESCRT-III. PLOS Comput Biol. 2009;5(11):e1000575.

16. Chiaruttini N, Roux A. Dynamic and elastic shape transitions in curved ESCRT-IIl filaments. Curr Opin Cell Biol. 2017;47:126-35. Cell Organelles. Available from: http://www.sciencedirect.com/science/article/pii/ S0955067417300935.

17. Agudo-Canalejo J, Lipowsky R. Domes and cones: adhesion-induced fission of membranes by ESCRT proteins. PLoS Comput Biol. 2018;14(8): e1006422.

18. Chiaruttini N, Redondo-Morata L, Colom A, Humbert F, Lenz M, Scheuring S, et al. Relaxation of loaded ESCRT-III spiral springs drives membrane deformation. Cell. 2015;163(4):866-79.

19. Pires R, Hartlieb B, Signor L, Schoehn G, Lata S, Roessle M, et al. A crescent-shaped ALIX dimer targets ESCRT-III CHMP4 filaments. Structure. 2009;17(6):843-56.

20. Ghazi-Tabatabai S, Saksena S, Short JM, Pobbati AV, Veprintsev DB, Crowther RA, et al. Structure and disassembly of filaments formed by the ESCRT-III subunit Vps24. Structure. 2008;16(9):1345-56.

21. Yuan H, Huang C, Li J, Lykotrafitis G, Zhang S. One-particle-thick, solvent-free, coarse-grained model for biological and biomimetic fluid membranes. Phys Rev E. 2010;82:011905. Available from: https://link.aps. org/doi/10.1103/PhysRevE.82.011905.

22. Cooke IR, Kremer K, Deserno M. Tunable generic model for fluid bilayer membranes. Phys Rev E. 2005;72(1):011506.

23. Plimpton S. Fast parallel algorithms for short-range molecular dynamics. J Comput Phys. 1995;117(1):1-19. Available from: http://www. sciencedirect.com/science/article/pii/S002199918571039X. 
24. Curk T, Wirnsberger P, Dobnikar J, Frenkel D, Šarić A. Controlling cargo trafficking in multicomponent membranes. Nano Lett. 2018;18(9):5350-6.

25. Stukowski A. Visualization and analysis of atomistic simulation data with OVITO - the Open Visualization Tool. Model Simul Mater Sci Eng. 2010;18(1):015012. Available from: http://stacks.iop.org/0965-0393/18/i= $1 / a=015012$.

26. Paraschiv A, Hegde S, Ganti R, Pilizota T, Šarić A. Dynamic clustering regulates activity of mechanosensitive membrane channels. bioRxiv. 2019553248. https://doi.org/10.1101/553248.

27. McMillan BJ, Tibbe C, Drabek AA, Seegar TC, Blacklow SC, Klein T. Structural basis for regulation of ESCRT-III complexes by Lgd. Cell Rep. 2017;19(9):1750-7.

28. Tang S, Henne WM, Borbat PP, Buchkovich NJ, Freed JH, Mao Y, et al. Structural basis for activation, assembly and membrane binding of ESCRT-III Snf7 filaments. Elife. 2015;4:e12548.

29. Shen QT, Schuh AL, Zheng Y, Quinney K, Wang L, Hanna M, et al. Structural analysis and modeling reveals new mechanisms governing ESCRT-III spiral filament assembly. J Cell Biol. 2014;206(6):763-77.

30. Hanson Pl, Roth R, Lin Y, Heuser JE. Plasma membrane deformation by circular arrays of ESCRT-III protein filaments. J Cell Biol. 2008;180(2): 389-402.

31. Lata S, Schoehn G, Jain A, Pires R, Piehler J, Góttlinger HG, et al. Helical structures of ESCRT-III are disassembled by VPS4. Sci (NY). 2008;321(5894): 1354-7.

32. Dobro MJ, Samson RY, Yu Z, McCullough J, Ding HJ, Chong PLG, et al. Electron cryotomography of ESCRT assemblies and dividing Sulfolobus cells suggests that spiraling filaments are involved in membrane scission. Mol Biol Cell. 2013;24(15):2319-27.

33. Goliand I, Adar-Levor S, Segal I, Nachmias D, Dadosh T, Kozlov MM, et al. Resolving ESCRT-III spirals at the intercellular bridge of dividing cells using 3D STORM. Cell Rep. 2018;24(7):1756-64.

34. Pfitzner AK, Mercier V, Roux A. Vps4 triggers sequential subunit exchange in ESCRT-III polymers that drives membrane constriction and fission. bioRxiv. 2019718080. https://doi.org/10.1101/718080.

35. Teis D, Saksena S, Judson BL, Emr SD. ESCRT-II coordinates the assembly of ESCRT-III filaments for cargo sorting and multivesicular body vesicle formation. EMBO J. 2010;29(5):871-83.

36. Morita E, Sandrin V, McCullough J, Katsuyama A, Hamilton IB, Sundquist WI. ESCRT-III protein requirements for HIV-1 budding. Cell Host Microbe. 2011;9(3):235-42.

37. Schöneberg J, Lee IH, Iwasa JH, Hurley JH. Reverse-topology membrane scission by the ESCRT proteins. Nat Rev Mol Cell Biol. 2017;18(1):5.

38. Henne WM, Stenmark H, Emr SD. Molecular mechanisms of the membrane sculpting ESCRT pathway. Cold Spring Harb Perspect Biol. 2013;5(9):a016766.

39. Babst M, Katzmann DJ, Estepa-Sabal EJ, Meerloo T, Emr SD. Escrt-II: an endosome-associated heterooligomeric protein complex required for mvb sorting. Dev Cell. 2002;3(2):271-82. Available from: http://www. sciencedirect.com/science/article/pii/S1534580702002204.

40. Teis D, Saksena S, Emr SD. Ordered assembly of the ESCRT-III complex on endosomes is required to sequester cargo during MVB formation. Dev Cell. 2008;15(4):578-89.

41. Avalos-Padilla Y, Knorr RL, Javier-Reyna R, Garcia-Rivera G, Lipowsky R, Dimova $R$, et al. The conserved ESCRT-III machinery participates in the phagocytosis of Entamoeba histolytica. Front Cell Infect Microbiol. 2018;8: 53. Available from: https://www.frontiersin.org/article/10.3389/fcimb. 2018.00053.

42. Schoeneberg J, Yan S, Righini M, Pavlin MR, Lee IH, Carlson LA, et al. ATP-dependent force generation and membrane scission by ESCRT-III and Vps4. bioRxiv. 2018262170. https://doi.org/10.1126/science.aat1839.

43. Maity S, Caillat C, Miguet N, Sulbaran G, Effantin G, Schoehn G, et al. VPS4 triggers constriction and cleavage of ESCRT-III helical filaments. Sci Adv. 2019;5(4):eaau7198.

44. Franquelim HG, Khmelinskaia A, Sobczak JP, Dietz H, Schwille P. Membrane sculpting by curved DNA origami scaffolds. Nat Commun. 2018;9(1):811.

\section{Publisher's Note}

Springer Nature remains neutral with regard to jurisdictional claims in published maps and institutional affiliations.

Ready to submit your research? Choose BMC and benefit from:

- fast, convenient online submission

- thorough peer review by experienced researchers in your field

- rapid publication on acceptance

- support for research data, including large and complex data types

- gold Open Access which fosters wider collaboration and increased citations

- maximum visibility for your research: over $100 \mathrm{M}$ website views per year

At BMC, research is always in progress.

Learn more biomedcentral.com/submissions 